\title{
Basal cells of the human airways acquire mesenchymal traits in idiopathic pulmonary fibrosis and in culture
}

\author{
Hulda R Jonsdottir 1,2,3,8, Ari J Arason 1,2,3,8, Ragnar Palsson 1,2,4, Sigridur R Franzdottir 1,2, Tomas Gudbjartsson ${ }^{3,5}$, \\ Helgi J Isaksson ${ }^{4}$, Gunnar Gudmundsson ${ }^{6,7}$, Thorarinn Gudjonsson ${ }^{1,2}$ and Magnus K Magnusson 1,2,6
}

Idiopathic pulmonary fibrosis (IPF) is a progressive interstitial lung disease with high morbidity and mortality. The cellular source of the fibrotic process is currently under debate with one suggested mechanism being epithelial-to-mesenchymal transition (EMT) in the alveolar region. In this study, we show that airway epithelium overlying fibroblastic foci in IPF contains a layer of p63-positive basal cells while lacking ciliated and goblet cells. This basal epithelium shows increased expression of $\mathrm{CK} 14$, Vimentin and $\mathrm{N}$-cadherin while retaining E-cadherin. The underlying fibroblastic foci shows both $\mathrm{E}$ - and N-cadherin-positive cells. To determine if p63-positive basal cells were able to undergo EMT in culture, we treated VA10, a p63-positive basal cell line, with the serum replacement UltroserG. A sub-population of treated cells acquired a mesenchymal phenotype, including an $\mathrm{E}$ - to $\mathrm{N}$-cadherin switch. After isolation, these cells portrayed a phenotype presenting major hallmarks of EMT (loss of epithelial markers, gain of mesenchymal markers, increased migration and anchorage-independent growth). This phenotypic switch was prevented in p63 knockdown (KD) cells. In conclusion, we show that airway epithelium overlying fibroblastic foci in IPF lacks its characteristic functional identity, shows increased reactivity of basal cells and acquisition of a partial EMT phenotype. This study suggests that some p63-positive basal cells are prone to phenotypic changes and could act as EMT progenitors in IPF.

Laboratory Investigation (2015) 95, 1418-1428; doi:10.1038/labinvest.2015.114; published online 21 September 2015

Idiopathic pulmonary fibrosis (IPF) is a severe lung disease characterized by progressive diminution in lung function based on an underlying fibrotic process in the lung parenchyma. ${ }^{1}$ The characteristic fibrosis contains the so-called fibroblastic foci found in close proximity to the lung epithelium. Many studies have indicated the importance of the pulmonary epithelial cells as the initial cellular target of injury. ${ }^{2-4}$ Although critical steps in the progressive fibrosis have been delineated, there are still major questions unanswered, especially regarding the interaction between epithelium and fibroblastic foci and cellular origin of the myofibroblasts that dominate in fibrotic foci of IPF.

Different cellular sources have been suggested to contribute to the fibroblastic foci in IPF. These include resident fibroblasts/myofibroblasts from the lung, mesenchymal stem cells derived from blood that have taken residence within the lung parenchyma and lung epithelial cells that have undergone epithelial-to-mesenchymal transition (EMT). ${ }^{5}$
EMT is a process well known in normal development, wound healing and cancer metastasis. ${ }^{6-8}$ The initiation of EMT is commonly marked by repression of the epithelial cell adhesion molecule E-cadherin and the dissociation of epithelial cells. The epithelial cells lose their distinct marker expression and gain a mesenchymal expression profile and morphology. ${ }^{9}$ This expressional switch includes an increase in the expression of various mesenchymal markers, such as Vimentin, $\mathrm{N}$-cadherin and fibronectin. ${ }^{10}$

Recently, studies have shown that various microRNAs (miRNAs), predominantly the miR-200 family, have an important role in both EMT and lung fibrosis. ${ }^{11,12}$ The miR-200 family member miR-200c is believed to be pivotal when it comes to the downregulation of E-cadherin, one of the hallmarks of EMT. ${ }^{13}$ When this miRNA is downregulated, its target, the transcription factor ZEB1, is free to downregulate E-cadherin expression. ${ }^{14}$

${ }^{1}$ Stem Cell Research Unit, Biomedical Center, Faculty of Medicine, University of Iceland, Reykjavik, Iceland; ²Department of Laboratory Hematology, Landspitali University Hospital, Reykjavik, Iceland; ${ }^{3}$ Faculty of Medicine, University of Iceland, Reykjavik, Iceland; ${ }^{4}$ Department of Pathology, Landspitali University Hospital, Reykjavik, Iceland;

${ }^{5}$ Department of Cardiothoracic Surgery, Landspitali University Hospital, Reykjavik, Iceland; ${ }^{6}$ Department of Respiratory Medicine and Sleep, Landspitali University Hospital, Reykjavik, Iceland and ${ }^{7}$ Department of Pharmacology and Toxicology, Faculty of Medicine, University of Iceland, Reykjavik, Iceland

Correspondence: Professor MK Magnusson, MD, Department of Pharmacology and Toxicology, Faculty of Medicine, University of Iceland, Vatnsmyrarvegur 16, Reykjavik 101, Iceland.

E-mail: magnuskm@hi.is

${ }^{8}$ These authors contributed equally to this work.

Received 2 December 2014; revised 17 July 2015; accepted 29 July 2015 
To argue against EMT as a driver of fibrosis in IPF, it has been shown that in the bleomycin-induced mouse model of pulmonary fibrosis multiple stromal cell populations contributed to the pulmonary fibrosis without any evidence for EMT. ${ }^{15}$ A more recent study suggested pathophysiological differences between human IPF and the bleomycin-induced mouse model and furthermore, suggested that the transcription factor GRHL2 may have a crucial role in epithelial activation in lung fibrosis and perhaps also in epithelial plasticity. ${ }^{16}$

Basal cells reside in the airway epithelium from the trachea and, in diminishing numbers, down to the respiratory bronchioles. ${ }^{17}$ They are candidate stem cells in the conducting airways, responsible for normal cell replacement and epithelial remodeling upon lung injury. ${ }^{18}$ Upon damage in airway epithelium, the basal cells show increased reactivity demonstrated by increased expression of p63, a basal cell restricted transcription factor and its downstream target cytokeratin 14 (CK14). ${ }^{19,20}$ Recent studies have implicated p63 with EMT. Das et al ${ }^{21}$ showed that fibrotic lesions in oral submucosa showed marked upregulation of p63 and Oh et $a^{22}$ showed that primary human keratinocytes transfected with $\Delta \mathrm{Np} 63$ underwent EMT when they had depleted their normal proliferative capacity.

Alveolar type II cells have been suspected as the cellular source of IPF through EMT. ${ }^{2,4}$ However, the contribution of basal cells has also been implicated. Chilosi et al ${ }^{23}$ showed highly increased numbers of p63-positive cells in bronchioles of IPF lungs, at areas showing both epithelial hyperplasia and squamous metaplasia. Seibold et $a^{24}$ found bronchial basal-, ciliated- and goblet cells to populate the characteristic honeycomb cysts in IPF lungs. However, the possible connection between basal cells and the EMT process in IPF still remains an open question. One reason for the lack of current understanding of the cellular origin of IPF is the lack of representing cell culture models to study diseases progression.

We have recently generated a bronchial epithelial cell line referred to as VA10. VA10 has basal cell properties evidenced by p63 and CK14 expression and its ability to generate pseudostratified epithelium in air-liquid interface (ALI) culture. ${ }^{25}$ In addition, this cell line forms bronchio-alveolarlike structures in three-dimensional co-culture with endothelial cells. ${ }^{25,26}$ In this study, we focus on the p63-positive lung epithelial basal cell as one potential progenitor cell for the myofibroblast in IPF.

We present data demonstrating that the epithelial cells in airways adjacent to fibroblastic foci show evidence of partial mesenchymal differentiation. Furthermore, using the VA10 in vitro basal cell model, we show that basal cells are able to undergo EMT, evidenced by both expression pattern, as well as cellular phenotypic characterization and that this transition is dependent upon p63 being present in the cells.

\section{MATERIALS AND METHODS}

\section{Ethics Statement}

Lung tissue samples were provided by written informed consent from patients who underwent open lung surgical procedures. This study was approved by the Landspitali Hospital Ethics Committee. Reference number 88374-96345.

\section{Samples of Lung Tissue from Patients with IPF}

Samples were analyzed from eight patients who underwent open lung biopsy at the Department of Cardiothoracic Surgery at Landspitali University Hospital. In all cases, the surgical biopsy was obtained with video-assisted thoracoscopic surgical approach that has been described elsewhere. ${ }^{27}$ These patients fulfilled criteria for having IPF as judged by a multidisciplinary clinical team. Sections that showed clear fibroblastic foci were selected. Control samples were obtained from patients who had undergone lobectomies and were free of pulmonary fibrosis.

\section{Cell Culture}

The bronchial epithelial cell line VA10 was previously established at the laboratory. ${ }^{25}$ VA10 cells were cultured in LHC-9 medium (Invitrogen, NY, USA) supplemented with $50 \mathrm{IU} / \mathrm{ml}$ penicillin and $50 \mathrm{mg} / \mathrm{ml}$ streptomycin (Invitrogen). UltroserG-treated VA10 cells were cultured in DMEM/F12 (Invitrogen) supplemented with 2\% UltroserG (Pall Biosepra, France) and $50 \mathrm{IU} / \mathrm{ml}$ penicillin and $50 \mathrm{mg} / \mathrm{ml}$ streptomycin (Invitrogen). 3D cultures were performed as previously described. ${ }^{26}$

\section{Production of Lentivirus and Cell Transduction}

Lentiviral transduction was performed as previously described. ${ }^{28}$ In short, plasmids containing scrambled hairpin (pLKO.1 shSCR; Addgene plasmid 17920) ${ }^{29}$ or shRNA against p63 (shp63alpha pLKO.1 puro; Addgene plasmid 19120) ${ }^{30}$ were used with packaging plasmids (psPAX2 and pMD2.G) (Addgene plasmids 12260 and 12259, respectively) to generate viral titer in HEK-293 $\mathrm{T}$ cells using Arrest-in (Open Biosystems). Transduction of VA10 cells was performed using low MOI volume and $8 \mathrm{mg} / \mathrm{ml}$ polybrene and positive cells selected with $0.7 \mu \mathrm{g} / \mathrm{ml}$ puromycin.

\section{Cell Sorting}

VA10 cells were sorted using Magnetic Cell Sorting (MACS, Miltenyi Biotec, Germany). Cells were suspended in MACS buffer (Miltenyi Biotec) and incubated, either with primary microbeads for EpCAM (CD326, Miltenyi Biotec) or unconjugated primary antibody for Thy-1 (clone AS02) for $30 \mathrm{~min}$ at $4{ }^{\circ} \mathrm{C}$. Unbound beads or antibody were removed by three consecutive washes with MACS buffer. For primary antibodies, a secondary incubation with anti-mouse IgG microbeads (Miltenyi Biotec) was conducted for $30 \mathrm{~min}$ at $4{ }^{\circ} \mathrm{C}$. After incubation, cells were washed three times with MACS buffer. Cells were then suspended in $1 \mathrm{ml}$ of MACS buffer and either loaded onto manual MACS separation 
columns (MS or LD columns, Miltenyi Biotec) or run through an autoMACS cells sorter (Miltenyi Biotec).

\section{Migration Assay}

A total of 20000 starved VA10 cells (DMEM only, $24 \mathrm{~h}$ ) were seeded onto migration filters ( $8 \mu \mathrm{m}$ pores, BD Bioscience) in DMEM (Invitrogen) supplemented with $50 \mathrm{IU} / \mathrm{ml}$ penicillin and $50 \mathrm{mg} / \mathrm{ml}$ streptomycin (Invitrogen). Endothelial growth medium (LONZA) supplemented with 5\% FBS (Invitrogen) was then added to the lower well. Cells were allowed to migrate for $24 \mathrm{~h}$. Then fixed for $5 \mathrm{~min}$ in $3.5 \%$ formaldehyde (Sigma Aldrich) and stained with $0.1 \%$ crystal violet for 15 min. Filters were rinsed with PBS and cells on the apical layer of the filter wiped off with a Q-tip. Density of migrated cells was evaluated by dissolving migrated cells in $0.5 \mathrm{ml} 10 \%$ acetic acid (Sigma Aldrich) and measuring optical density at $590 \mathrm{~nm}$ using a spectrometer.

\section{Soft Agar Assay}

Plates were coated with $1 \%$ soft agar (Sigma Aldrich) diluted in DMEM (Invitrogen) and kept at $4{ }^{\circ} \mathrm{C}$ for 20 min to allow solidification. A total of 30000 cells were seeded into $0.5 \%$ soft agar preheated to $40{ }^{\circ} \mathrm{C}$ and placed onto the solidified $1 \%$ surface. The plate was incubated at $4^{\circ} \mathrm{C}$ for $10 \mathrm{~min}$ to allow solidification of the upper layer. Cells were then cultured for 20 days. After 20 days, cells were stained with $0.05 \%$ crystal violet for $1 \mathrm{~h}$ and colonies over $30 \mu \mathrm{m}$ in size were counted in each well using a phase contrast microscope (Leica). Three representative areas were counted in each well. Each experiment was conducted in triplicate.

\section{RNA Isolation, CDNA Synthesis and qRT-PCR}

RNA was isolated from VA10 cells using TriReagent solution (Sigma Aldrich). Reverse transcription was carried out using the RevertAid First Strand Synthesis Kit (Fermentas) according to the manufacturer's instructions. For quantification of miR-200c, hsa-miR-200c-3p LNA ${ }^{\mathrm{TM}}$ PCR primer set (Exicon, 204482) was used with an 3 ' non-fluorescent MGB quencher and FAM 5' reporter dye. GAPDH was used as the endogenous reference gene and amplified using commercially available primers (Applied Biosystems, 4326317E). Data were analyzed using 7500 Software v2.0 (Applied Biosystems).

\section{Immunochemistry}

Paraffin-embedded tissue samples of control and IPF lung biopsies were obtained from the Department of Pathology, Landspitali University Hospital. The samples were deparaffinized, antigen retrieved by boiling in TE buffer for $20 \mathrm{~min}$ (unless stated otherwise) and stained with EnVisionH+ System-HRP kit (Dako) according to the manufacturer's instructions. Primary antibodies (see below) incubated at RT for $30 \mathrm{~min}$. Cell cultures were fixed with $3.5 \%$ fomaldehyde for $10 \mathrm{~min}$ at RT or with methanol at $-20^{\circ} \mathrm{C}$. Cultures were then washed two times with PBS and then blocked with $10 \%$ FBS in IF buffer $(0.2 \%$ Triton X-100; $0.1 \%$ BSA and $0.05 \%$
Tween-20 in PBS). Primary antibodies incubated for $30 \mathrm{~min}$ at RT followed by three 10-min washes with PBS. Secondary isotype-specific Alexa Fluor antibody conjugates (Invitrogen) were incubated for $30 \mathrm{~min}$ at RT. Nuclei stained for $15 \mathrm{~min}$ at RT using TO-PRO-3 (Invitrogen). Samples were embedded in Fluoromount-G (Southern Biotech, Birmingham, AL, USA) and analyzed with confocal microscopy (Zeiss LSM5 Pasqal). Representative confocal images were quantified using CellProfiler. ${ }^{31}$

\section{Western Blot}

In all, $5 \mu \mathrm{g}$ of protein lysate was mixed with $4 \mathrm{X}$ NuPage LDS sample buffer (Invitrogen), reduced with mercaptoethanol (Sigma Aldrich) and heated to $75^{\circ} \mathrm{C}$ for $10 \mathrm{~min}$. Then loaded on $10 \%$ SDS Bis-Tris gels (Invitrogen) and run on $180 \mathrm{~V}$ for $45 \mathrm{~min}$ in $1 \mathrm{X}$ NuPage MES running buffer (Invitrogen). Proteins were transferred at $30 \mathrm{~V}$ for $1.5 \mathrm{~h}$ in $1 \mathrm{X}$ NuPage transfer buffer (Invitrogen) containing 10\% methanol to a methanol-activated (Sigma Aldrich) PVDF membrane (Millipore). After transfer, membrane was washed in PBS at room temperature for $5 \mathrm{~min}$ and then blocked with 5\% BSA (Applichem) in TBS+0.1\% Tween (Sigma Aldrich) for $1 \mathrm{~h}$. Primary antibodies were incubated overnight at $4{ }^{\circ} \mathrm{C}$ in $5 \%$ BSA in $0.1 \%$ TBS+Tween. The next day, the membrane was washed for $3 \times 10 \mathrm{~min}$ in PBS. Secondary infrared (IR) antibodies (LiCOR) were incubated in $0.1 \%$ TBS-Tween $+0.02 \%$ SDS (Sigma Aldrich) for $1 \mathrm{~h}$ at 1:20 000 dilution. Subsequently, the membrane was washed for $3 \times 10 \mathrm{~min}$ in PBS. Signal detection and analysis were performed using an Oddysey Infrared Image Scanner and the corresponding Image Studio software (LiCOR).

\section{List of antibodies}

\begin{tabular}{|c|c|c|c|c|c|c|}
\hline Antibody & Species & Clone & $\#$ & Manufacturer & Dilution & Application \\
\hline E-cadherin & Mouse & 34 & 610182 & $\mathrm{BD}$ & 1:100/1000 & Tissue/WB \\
\hline $\mathrm{N}$-cadherin & Mouse & 32 & 610921 & $\mathrm{BD}$ & 1:100/1000 & Tissue/WB \\
\hline EpCAM & Mouse & VU-1D9 & ncl-ESA & Leica & 1:100/1000 & IF/WB \\
\hline Vimentin & Mouse & V9 & M0725 & Dako & $\begin{array}{c}1: 100 / 100 / \\
1000\end{array}$ & Tissue/IF/WB \\
\hline CK14 & Mouse & LLL02 & ncl-I-II002 & Leica & $1: 100$ & Tissue/IF \\
\hline Thy-1 & Mouse & ASO2 & CP28 & Calbiochem & $\begin{array}{c}1: 100 / 1000 / \\
100\end{array}$ & IF/WB/CS \\
\hline p63 & Mouse & 7JUL & ncl-p63 & Leica & $1: 25 / 500$ & Tissue/WB \\
\hline Actin & Mouse & $C 4$ & 3280 & Abcam & $1: 2000$ & WB \\
\hline Fibronectin & Mouse & & N/A & $\begin{array}{l}\text { Prof. Deane } \\
\text { Mosher lab }\end{array}$ & $1: 200$ & IF \\
\hline
\end{tabular}

\section{Statistical Analysis}

Data are presented as mean and s.d. (error bars) from number of independent experiments. Graphs and calculations 
were done using GraphPad Prism. All experimental procedures were repeated at least three times. All samples were tested for equal variance.

\section{RESULTS}

\section{Airway Epithelium Overlying Fibroblastic Foci in IPF Has Lost Pseudostratified Epithelial Architecture and Acquired a Partial Mesenchymal Phenotype}

To analyze the phenotype of the airway epithelium in IPF, we stained lung tissue from IPF patients and controls with markers of various epithelial and mesenchymal differentiation stages. We found that in IPF patients, CK14, a marker known to be upregulated during lung tissue repair, ${ }^{32}$ is strongly expressed in the bronchial epithelium adjacent to fibroblastic foci (Figure 1). Another basal cell marker, the transcription factor p63, a known regulator of CK14 expression showed increased expression that follows a similar pattern as CK14. No expression of CK14 and p63 was found within the fibroblastic foci. Interestingly, the epithelium shows a more stratified squamous phenotype around the foci with CK14 and p63 expression also found in suprabasal cells (Figure 1 and Supplementary Figure S1). We found it to be a general rule of thumb that airway epithelium adjacent to a focus showed this phenotype. E-cadherin was strongly expressed in all epithelial cells, but interestingly, cells within the fibroblastic foci also stained positive, whereas E-cadherin expression in control samples is limited to the epithelium (Figure 1). To further analyze whether the epithelial cells had acquired a mesenchymal phenotype, we stained for $\mathrm{N}$-cadherin, a classical marker for EMT. N-cadherin is highly expressed during human lung development but in the normal adult human lung $\mathrm{N}$-cadherin is primarily expressed in mesothelial cells and scattered basal cells in bronchioles. ${ }^{33}$ Interestingly, basal cells close to fibroblastic foci showed increased $\mathrm{N}$-cadherin expression compared with control samples. When we stained for the mesenchymal marker Vimentin, strong expression was seen in the stroma, including the sub-epithelial fibroblastic foci. Expression of Vimentin was also highly upregulated in epithelium adjacent to fibroblastic foci.

Figure 1 Basal cell reactivity, hyperplasia and upregulation of EMT markers adjacent to fibroblastic foci. Epithelium adjacent to fibroblastic foci shows increased reactivity and mesenchymal phenotype. Sparse staining of CK14 is seen in control samples, whereas CK14 is strongly expressed in epithelium overlying fibroblastic foci. P63, a restricted transcription factor for basal cells is expressed in epithelial cells located both basally and in the top layer indicating formation of metaplasia in IPF samples. E-cadherin is abundant in epithelium both in control and IPF samples. In addition, E-cadherin is present in cells within the foci. N-cadherin is expressed in lower basal epithelial cells in IPF samples whereas control samples are negative. Vimentin is expressed in both epithelium and mesenchyme in IPF samples. Bar $100 \mu \mathrm{m}$.

\section{Phenotypic Plasticity of Bronchial-Derived Basal Epithelial Cells in Culture}

Given the mixed mesenchymal and epithelial differentiation pattern in the bronchial epithelium adjacent to fibroblastic foci and the presence of E-cadherin-positive cells within the foci, we wanted to evaluate whether bronchial-derived basal cells could undergo phenotypic changes toward EMT, thus

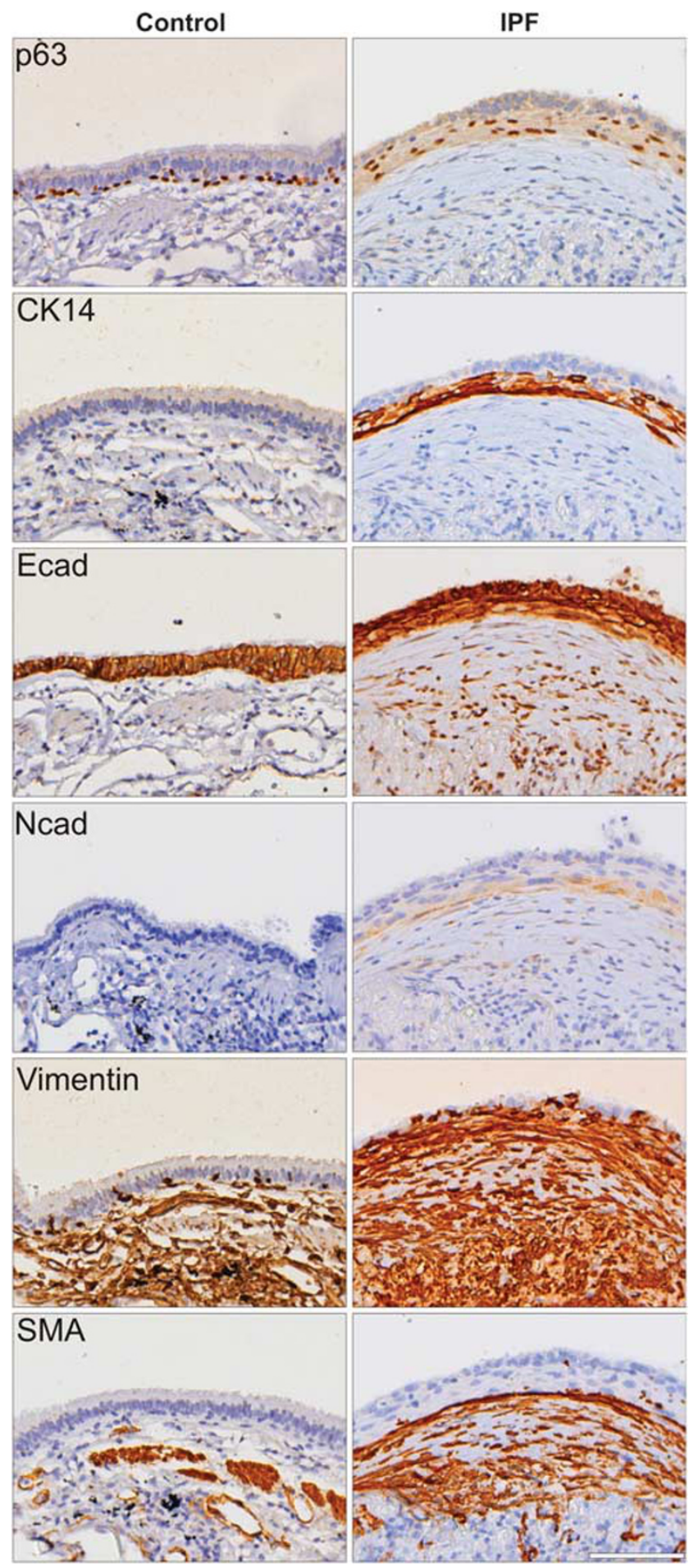


contributing to the fibrotic process of pulmonary fibrosis. We have previously generated a human bronchial-derived basal epithelial cell line (VA10) that is capable of generating a pseudostratified epithelial layer in ALI cultures. ${ }^{25}$ In addition, VA10 forms bronchioalveolar-like structures in 3D co-culture with endothelial cells ${ }^{26}$ demonstrating the stem cell-like properties of this cell line. To analyze the plasticity of cells with basal cell properties, we tested if VA10 cells could be

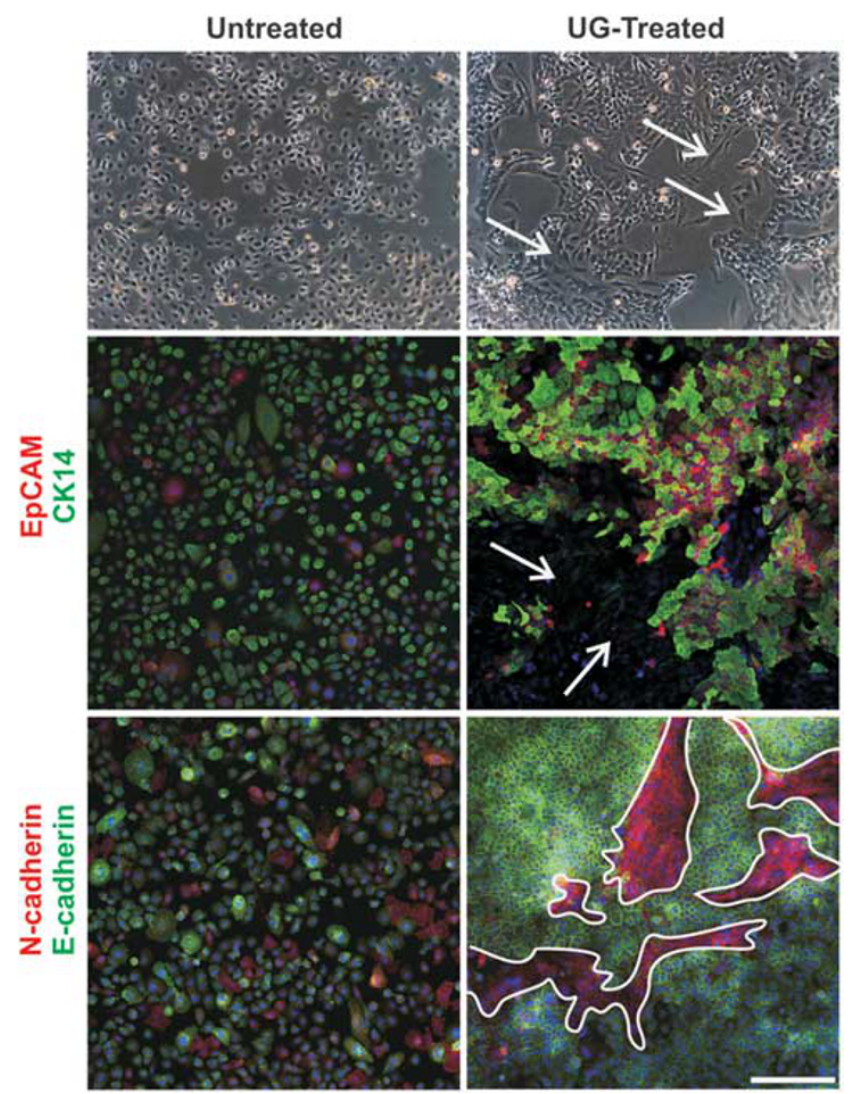

Figure 2 UltroserG induces epithelial-to-mesenchymal transition in a subset of VA10 cells. Sub-population of cells cultured in UltroserG medium (UG-treated) show elongated phenotype (top panel, arrows). EpCAM and CK14 immunostaining reveals an EpCam/CK14-negative mesenchymal sub-population in treated cells (middle panel, arrows). Double staining for $\mathrm{E}$ - and $\mathrm{N}$-cadherin also shows two distinct cellular sub-populations in treated cells indicating a cadherin switch (bottom panel, outline). Cells were counterstained with TOPRO-3 nuclear stain (middle and bottom panel). Bar $200 \mu \mathrm{m}$. induced to undergo EMT. In vivo the bronchial basal cell is steadily maintained and, furthermore, gives rise to daughter cells capable of undergoing mucociliary differentiation. This in vivo-like behavior can be mimicked in culture using one of several culture media and the right in vitro conditions. We selected the serum substitute UltroserG (UG) commonly used for ALI culture to facilitate differentiation of basal cells ${ }^{34,35}$ and a known inducer of EMT. ${ }^{36,37}$ In contrast to the cuboidal epithelial phenotype seen in monolayer when cultured in bronchial-defined medium (serum-free LHC-9 medium), a sub-population of VA10 cells in UG supplemented media (2\%) showed distinct changes toward a mesenchymal phenotype (Figure 2, top panel). In UG media, the VA10 cells arrange into two distinct sub-populations, islands of cuboidal epithelial-like cells separated by a zone of elongated fibroblast-like cells (Figure 2, top right: arrows). Immunophenotypic characterization revealed that the epithelial-like islands displayed an epithelial phenotype, expressing EpCAM, CK14 and E-cadherin. The elongated fibroblast-like cells, however, showed a mesenchymal phenotype as evidenced by reduced expression of EpCAM, CK14 and E-cadherin and acquisition of $\mathrm{N}$-cadherin expression (Figure 2, lower two panels: arrows and outline and Supplementary Figure S2). Interestingly, no phenotypic change is observed when VA10 cells are treated with transforming growth factor beta 1 (TGF- $\beta 1$ ), a well-known EMT inducer (data not shown). EpCAM is ubiquitously expressed in bronchial epithelium but its high expression has been linked to stem cell properties in number of epithelial tissues. ${ }^{38,39}$ Furthermore, downregulation of EpCAM is frequently observed in EMT. ${ }^{40,41}$ Therefore, we next decided to separate EpCAM-positive and EpCAMnegative cellular sub-populations and evaluate if these cells retained their epithelial and mesenchymal traits, respectively, and analyze the phenotypic plasticity of each population.

\section{Purification and Characterization of Mesenchymal Cells Derived from VA10}

Confluent VA10 cells cultured in UG media were magnetically separated (MACS) based on their EpCAM status. Further purification of EpCAM ${ }^{\text {neg }}$ cells was done by selecting for Thy-1-positive cells (flow-diagram, Figure 3a). VA10EpCAM $^{\text {pos }}$ (VA10E) demonstrated a cuboidal epithelial phenotype, whereas VA10-EpCAM ${ }^{\text {neg }} /$ Thy- $^{\text {pos }}$ (VA10M) showed elongated spindle-like phenotype (Figure 3c,

Figure 3 Purification and characterization of VA10-derived epithelial and mesenchymal sub-populations. (a) Purification of epithelial and mesenchymal sub-populations from VA10. Using immunomagnetic cell sorting against EpCAM VA10 was split into EpCAM ${ }^{\text {high }}$ (left) and EpCAM ${ }^{\text {low }}$ (right). The EpCAM $^{\text {low }}$ sub-population was further sorted according to Thy-1 expression. The resulting sublines will hereafter be termed VA10E and VA10M, respectively. (b) Surface marker expression of VA10M (Thy $-1^{+}$) indicates a true mesenchymal phenotype. Immunoblot analysis reveals a difference in epithelial and mesenchymal marker expression between the different cell lines tested. E-cadherin expression has been abolished in VA10M, whereas $\mathrm{N}$-cadherin and Vimentin are upregulated indicating a mesenchymal expression pattern. (c) Characterization of VA10-derived epithelial- and mesenchymal sub-populations. VA10E has a cobblestone-like morphology (top panel, left), whereas VA10M is elongated and fibroblast like (top panel, right). Immunostaining reveals a strong mesenchymal expression pattern in VA10M, whereas VA10E predominantly exhibits an epithelial phenotype (bottom panel). 
a

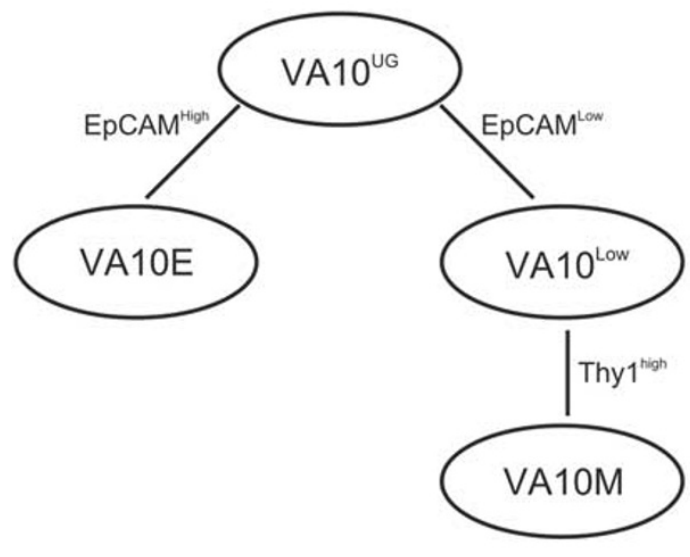

b
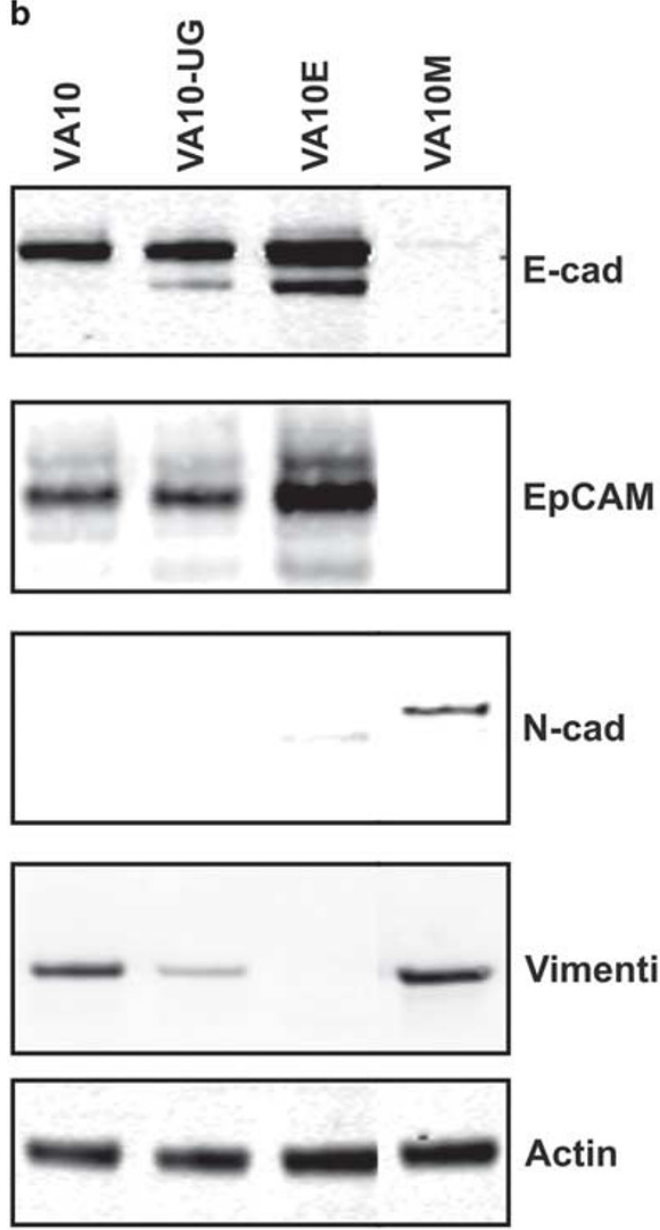

Vimentin

Actin c
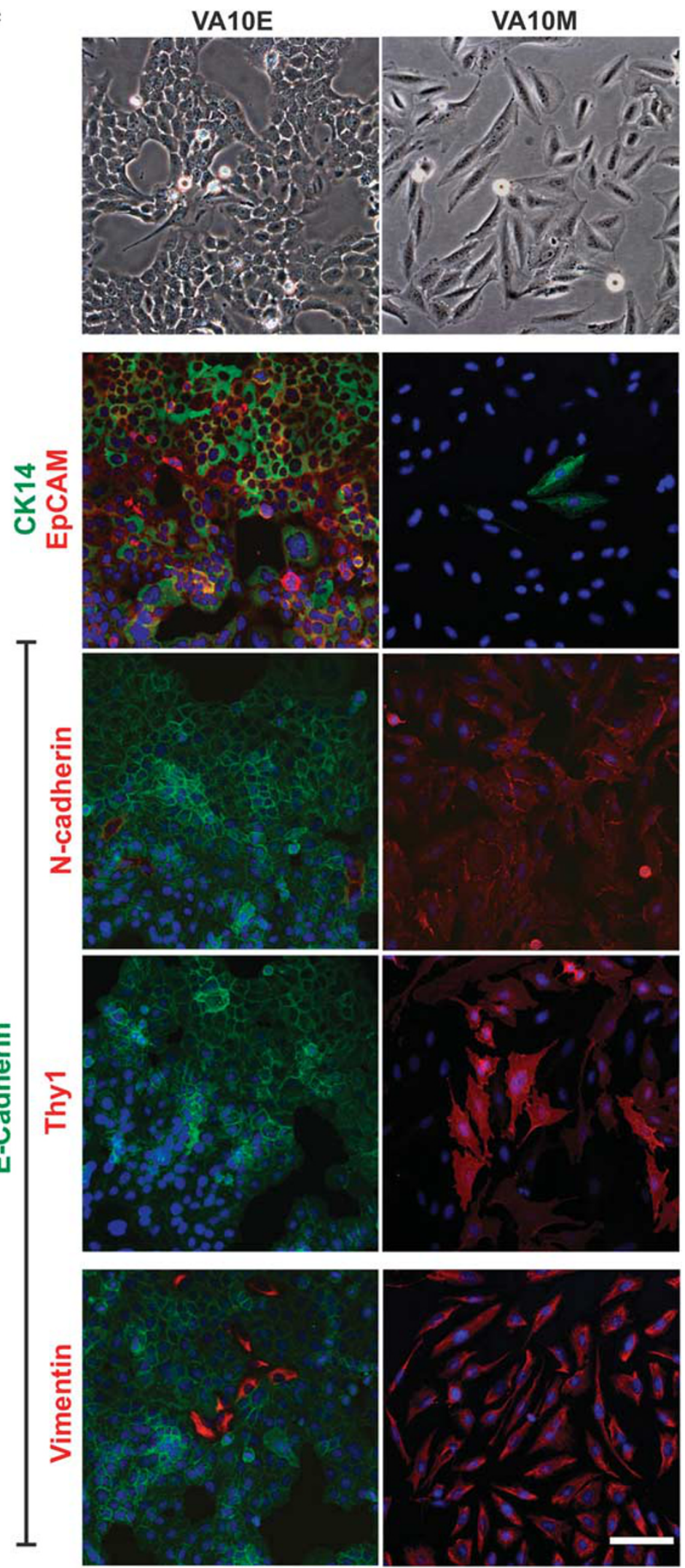
top panel). The epithelial and mesenchymal phenotype in VA10E and VA10M, respectively, were further evaluated with immunocharacterization (Figures $3 \mathrm{~b}$ and $\mathrm{c}$ ). Western blotting (Figure $3 \mathrm{~b}$ ) showed that untreated VA10 cells (in bronchial medium) and treated VA10 cells (in UG medium) showed strong expression of the epithelial markers E-cadherin and EpCAM. Expression of Vimentin was observed in both cell population but no expression of $\mathrm{N}$-cadherin was seen in VA10E. VA10E cells showed a strict epithelial phenotype, whereas VA10M cells were negative for both E-cadherin and EpCAM but positive for mesenchymal markers (Figure $3 b$ ). These phenotypic differences were further characterized and confirmed by immunofluorescence staining. VA10E cells were strongly positive for CK14, EpCAM and E-cadherin but negative for $\mathrm{N}$-cadherin, Thy-1. Vimentin expression can still be observed in a few cells (Figure $3 \mathrm{c}$, left). VA10M cells on the other hand had lost their epithelial phenotype except for occasional positive CK14 cells. VA10M were uniformly positive for the mesenchymal markers Thy-1, Vimentin and $\mathrm{N}$-cadherin (Figure $3 \mathrm{~b}$, right). Collectively, we have generated a mesenchymal sub-line from VA10 by exposing the cells to the serum substitute UG. The mesenchymal-like cells referred to as VA10M have undergone phenotypic changes suggestive of EMT.

\section{VA10M Has Acquired a Functional Mesenchymal Phenotype}

To further analyze the mesenchymal properties of VA10M, we conducted various functional assays that have been shown to associate with EMT. Migration, a phenotype associated with mesenchymal cells, was tested using a transwell migration assay. Although the VA10 and VA10E cell lines showed similar migratory capabilities, VA10M cells showed almost twofold more migration than the VA10E cells (Figure 4a). Anchorage-independent growth has also been linked with a mesenchymal phenotype and while both VA10 and VA10E cells show limited anchorage-dependent growth, the VA10M cells had three- to fourfold increased number of colonies compared with VA10E in a soft agar assay (Figure 4b).

A number of miRNAs, including the mir-200 family, has been shown to be important in the preservation of epithelial integrity. In particular, loss of miR-200c expression has been associated with loss of epithelial integrity and EMT. ${ }^{14}$ Although both VA10 and VA10E cells retain their miR-200c expression, its expression is markedly downregulated in VA10M. Also, in accordance to the miR-200c expression, upregulation of the mesenchymal EMT marker fibronectin was clearly detected in VA10M (Figure 4c). When cultured in a three-dimensional matrigel-based co-culture system with endothelial cells, both treated VA10 and VA10E cells form branching bronchioalveolar-like structures like the original VA10 cell line. ${ }^{26}$ Under these conditions, approximately $10 \%$ of colonies form large branching structures (Figure 4d, white bars). However, when VA10M cells were cultured in the same three-dimensional co-culture system they did not exhibit any branching but instead formed large spindle-like colonies similar to fibroblasts (Figure 4d, black bars). In all co-culture conditions, approximately $90 \%$ of colonies were small with no distinct morphology (Figure $4 \mathrm{~d}$, gray bars). These various functional assays show that although the VA10E sub-line retains the phenotype exhibited by its mother cell line (VA10), the VA10M cells have gained a markedly different phenotype, with clear mesenchymal properties. Interestingly, VA10M can be reverted back to the original phenotype by culturing the cells on LHC-9 medium (Supplementary Figure S3). In summary, we have shown that the p63-positive basal cell line VA10 is able, under specific conditions, to undergo EMT, both with regard to protein expression and cellular phenotype.

\section{KD of p63 Abrogates UG-Stimulated EMT Capacity of VA10}

To evaluate whether the expression of p63 is necessary for the mesenchymal transformation of VA10 cells, we performed a lentiviral-based stable KD with p63 and control scrambled shRNA in VA10 (VA10 $0^{\mathrm{p} 63 \mathrm{KD}}$ and $\mathrm{VA} 10^{\mathrm{SCR}}$, respectively). This resulted in a significant decrease in p63 protein levels (Supplementary Figure S4). When cultured in UG media, no spindle-like phenotype was observed in $\mathrm{VA} 10^{\mathrm{p} 63 \mathrm{KD}}$, compared with $\mathrm{VA} 10^{\mathrm{SCR}}$ (Figure 5). Immunostaining revealed that the $\mathrm{VA} 10^{\mathrm{p} 63 \mathrm{KD}}$ cells showed no upregulation of $\mathrm{N}$-cadherin or Thy-1, whereas retaining E-cadherin expression, in contrast to $\mathrm{VA} 10^{\mathrm{SCR}}$ (Figure 5). VA10 ${ }^{\mathrm{SCR}}$ showed more Vimentin expression in E-cadherin-negative cells, whereas Vimentin was exclusively co-expressed with E-cadherin in $\mathrm{KD}$ cells, indicating no sub-population distinction.

\section{DISCUSSION}

In this study, we have shown that basal epithelial cells overlying fibroblastic foci in IPF patient samples acquire increased reactivity as measured by p63 and CK14 expression and acquisition of a partial mesenchymal phenotype as measured by $\mathrm{N}$-cadherin and Vimentin expression. Although we cannot claim that basal cells are the cellular source of fibroblasts within the foci, there is no doubt that the overlying epithelium, in particular the basal cells, acquire mesenchymal traits that may be either the consequence of signals derived from the foci or signify that the epithelium is directly contributing to their formation by undergoing EMT. Furthermore, our in vitro studies show that the p63-positive basal cell line VA10 is able to undergo a mesenchymal transition as evidenced by induction of EMT upon culture in UG containing medium.

In the IPF samples, we see that the epithelium overlying the fibroblastic foci is rich in p63-positive cells that have not only a basal but also a suprabasal pattern and show a mixed E- and $\mathrm{N}$-cadherin expression along with both CK14 and Vimentin expression (Figure 1). This pattern is indicative of a partial EMT in the epithelial cells. Underlying this abnormal epithelium, there are abundant mesenchymal cells forming 
a

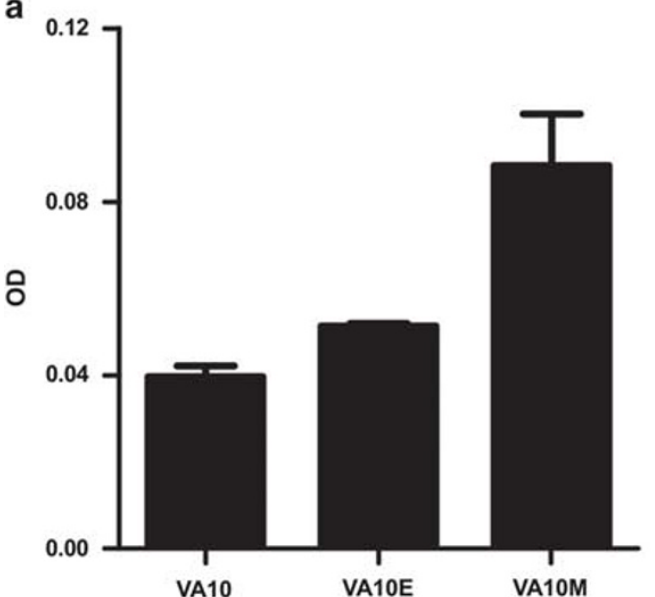

b

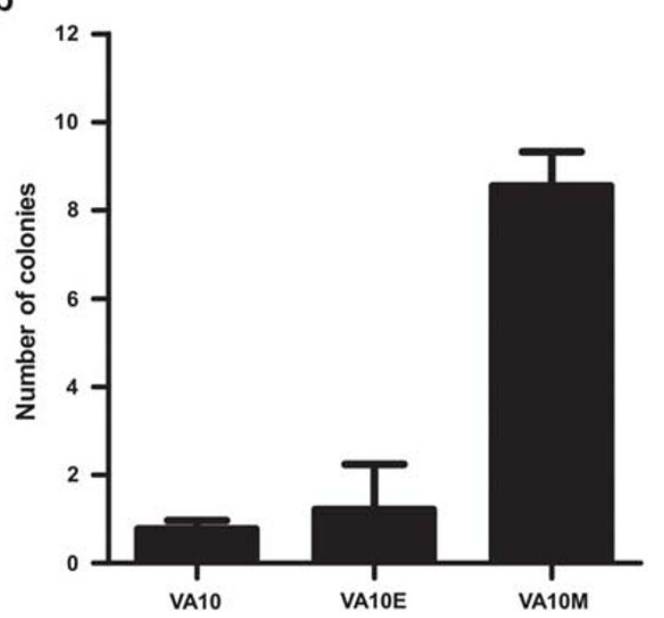

C
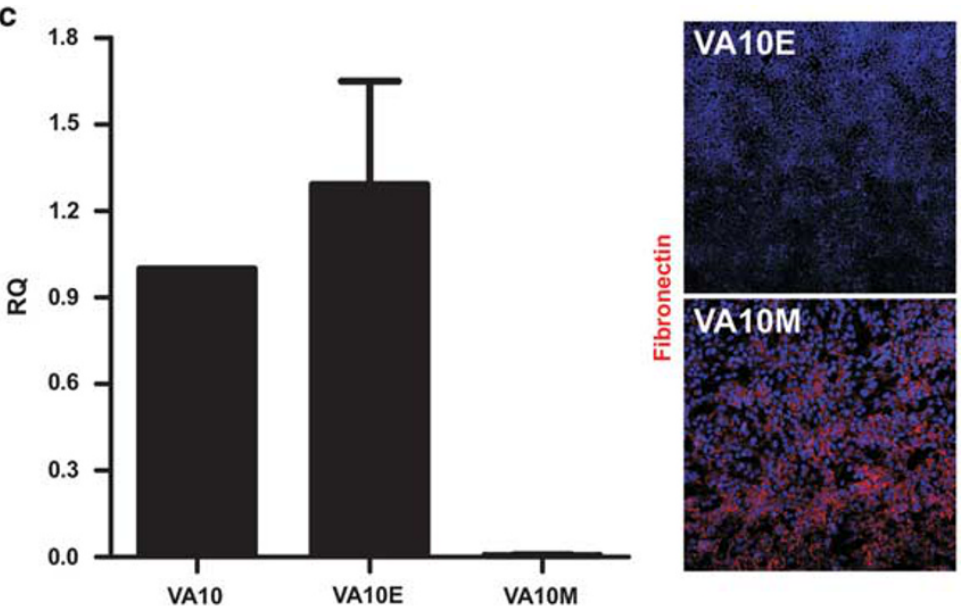

d

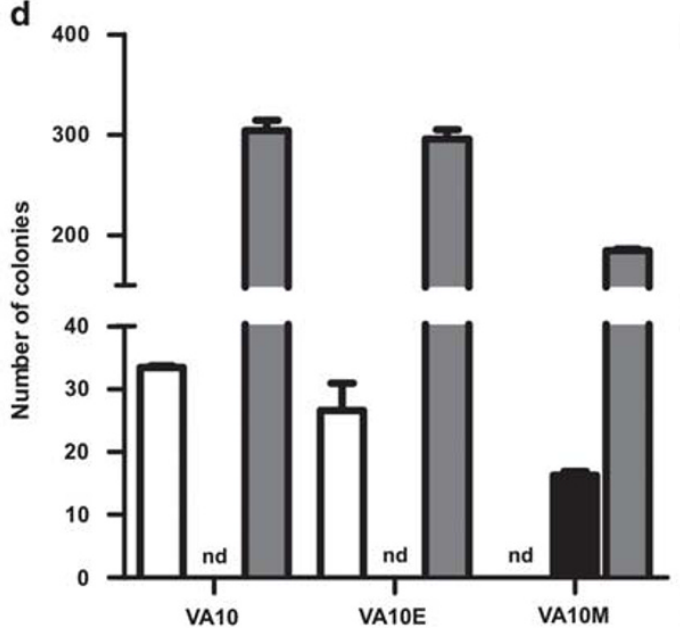

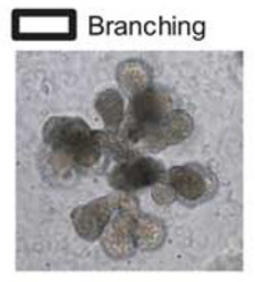

Spindle

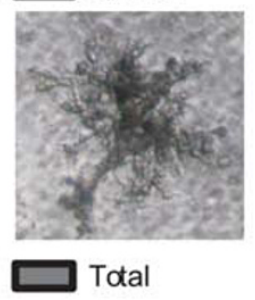

Figure 4 Functional characterization of VA10M. (a) VA10M cells have increased migratory potential compared with VA10E. VA10M cells migrate more actively than VA10E cells measured by migration through a porous filter and represented as optical density. (b) VA10M exhibits increased anchorageindependent growth. When cultured in soft agar VA10M cells show a higher potential for anchorage-independent growth than both UltroserG-treated VA10 and VA10E. (c) Expression of miRNA 200c is reduced in VA10M, whereas fibronectin expression is upregulated. qRT-PCR analysis reveals a loss of microRNA 200c expression in VA10M. As a result fibronectin is upregulated in VA10M, whereas it is absent in VA10E. (d) VA10M cells form spindle colonies in $3 \mathrm{D}$ cultures. When cultured in three-dimensional reconstituted basement membrane VA10 cells form branching colonies (roughly $10 \%$ of total colony number). Although VA10E follows the same pattern VA10M does not form any branching colonies under these conditions. The mesenchymal sub-population does, however, form spindle colonies in 3D cultures characteristic of cells having undergone EMT.

the fibroblastic foci but we can also see occasional E-cadherin-positive cells within the foci. This is an interesting pattern with abnormal p63-positive epithelial cells with signs of partial EMT overlying the mesenchymal-rich fibroblastic foci. This pattern thus raises the question whether the epithelium, specifically the p63-positive cells could contribute to the fibroblastic foci through EMT. Double positive CK14/ p63-positive basal cells are rare in the airway and such cells have been associated with injury or wound-healing responses. ${ }^{42}$ Interestingly, Oh et $\mathrm{al}^{22}$ have shown that primary human keratinocytes transfected with $\Delta \mathrm{Np} 63$ underwent EMT when they had depleted their normal proliferative capacity. Thus, injury-response CK14/p63-positive cells in the epithelium overlying the fibroblastic foci could be a cellular source for EMT.
Up to $20 \%$ of IPF cases have been suggested to be familial, often referred to as familial interstitial pneumonia (FIP). ${ }^{43}$ Conventional genetic approaches have identified four genes that are involved in FIP. These four genes encode proteins in two separate functional families: surfactant proteins, surfactant protein C $\left(\right.$ SFTPC) ${ }^{44}$ and surfactant protein A2 (SFTPA2), ${ }^{45}$ and components of the telomerase complex, telomerase reverse transcriptase (TERT) and the RNA component of telomerase (TERC). ${ }^{46,47}$ More recently, Schwartz and colleagues have, through several publications, linked a common single-nucleotide polymorphism in the promoter region of the mucin gene MUC5B to idiopathic interstitial pneumonias, including IPF. ${ }^{48-50}$

The mutations in the surfactant family proteins, SFTPC and SFTPA2, both expressed exclusively by type II alveolar 


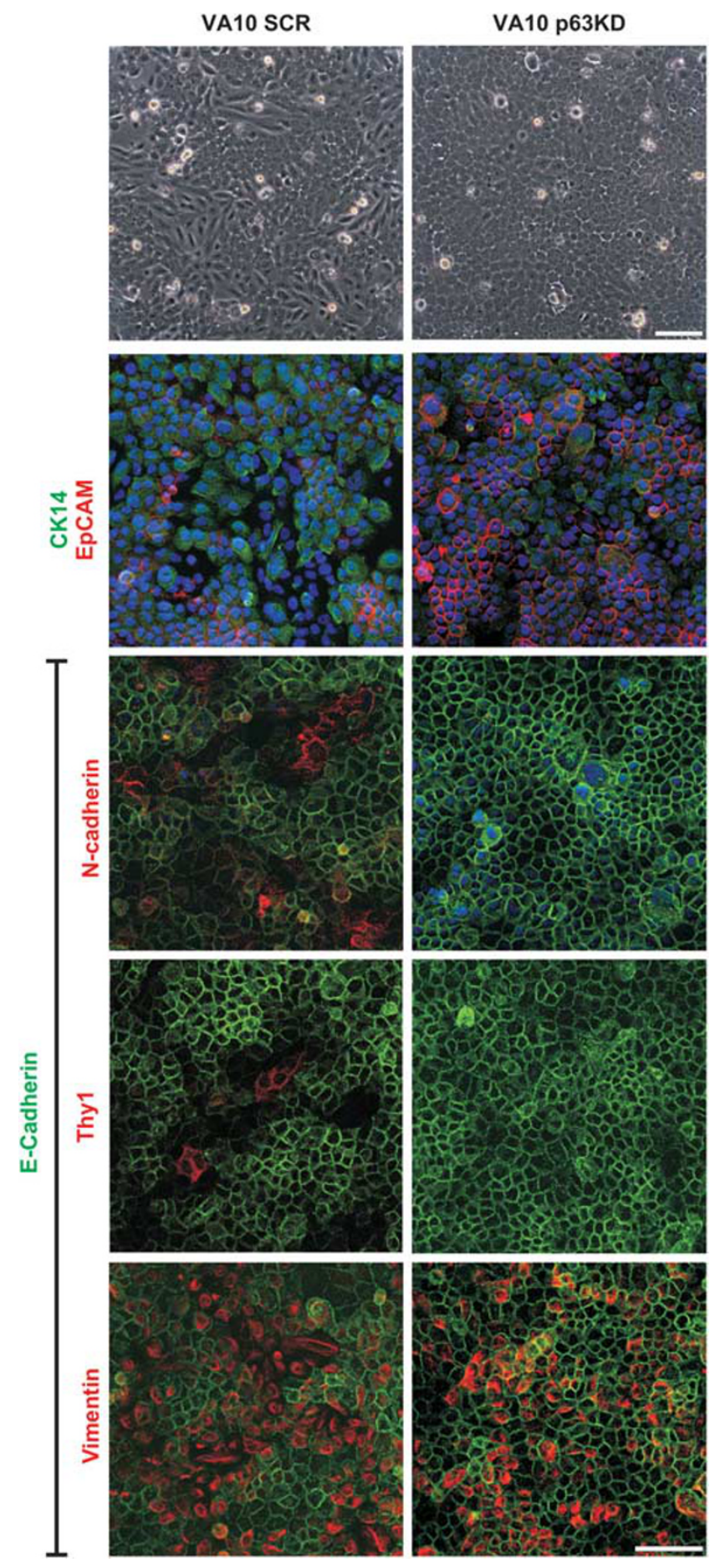

Figure 5 Knockdown (KD) of p63 abrogates UG-induced EMT of VA10 cells. p63 KD cells are unable to undergo UG-induced EMT. The expression of the mesenchymal markers $\mathrm{N}$-cadherin and Thy- 1 is abrogated in treated KD cells, whereas scrambled control cells show a division into two cellular sub-populations much like the mother cell line VA10. The expression of Vimentin can still be detected in treated KD cells, although to a lesser extent than in treated scrambled cells. Bars $100 \mu \mathrm{m}$. epithelial cells (AECs) in the lungs, suggest that AEC dysfunction is a prominent feature of IPF. Furthermore, accumulating evidence supports endoplasmic reticulum (ER) stress with consequent apoptosis as a prominent feature in IPF. Compared with COPD and normal lungs, protein levels of ER stress mediators, such as ATF-6, ATF-4 and the apoptosisinductor C/EBPzeta, were elevated in AECs of IPF lungs. ${ }^{51}$ How ER stress with subsequent epithelial injury leads to a fibrotic response in the adjacent mesenchymal compartment is still intensely debated. The two major hypotheses are that epithelial cells adjacent to the injured area undergo EMT or that the injury leads to proliferation of the local mesenchymal cells, such as fibroblasts or myofibroblasts. ${ }^{43}$ Although the surfactant type mutations have a described mechanism of epithelial injury through ER stress, the mechanism by which telomerase dysfunction (TERT and TERC mutations) leads to IPF is less understood. It is thought that epithelial cell dysfunction may be mediated by epithelial cell senescence and impaired response to epithelial injury, as a consequence of telomere dysfunction. ${ }^{52}$

Although most investigators agree that the epithelial compartment is where the initial injury occurs in IPF, there is much less known about the following events. As stated above, the main debate focuses on the origin of the cellular components of the fibrotic response. The progressive nature of the fibrotic scarring is what drives the destructive nature of IPF. The data collected with mouse models of surfactant mutants suggest that fibrosis in the commonly used bleomycin mouse model worsens in the background of ER stress. ${ }^{53}$ In the same bleomycin model with surfactant (SFTPC) mutated mice, the data also support the notion that ER stress can contribute to EMT in vivo. ${ }^{54}$ Overexpression of mutant SFTPC in lung epithelial cell lines largely recapitulated the ER stress-induced EMT. ${ }^{55}$ To argue against EMT as a driver of fibrosis, it has been shown as previously discussed that in the bleomcyininduced model multiple stromal cell populations contributed to the pulmonary fibrosis without any evidence for EMT. ${ }^{15}$

Another important unsolved issue is the epithelial cell of origin contributing to epithelial injury and EMT. The surfactant mutants would strongly suggest the AEC-type II cells, given their almost exclusive expression of surfactant proteins. This is also supported by the fact that the initial and main fibrotic changes in IPF are located distally in the airways. The possible contribution of the MUC5B promoter region polymorphism to IPF pathogenesis raises the question whether epithelia in upper airways may also contribute to IPF. In the normal lung, MUC5B is expressed in sub-mucosal cells of the upper airways, whereas goblet cells express MUC5B under stress conditions. ${ }^{56}$ In addition, TGF- $\beta 1$-induced EMT is a well-documented process in A549, an alveolar type II cell line. ${ }^{57}$ Interestingly, TGF- $\beta 1$ does not induce EMT in VA10 nor another bronchial-derived epithelial cell line, Calu-3. ${ }^{57}$ This indicates that the mechanism of EMT could be different in bronchial-derived cells compared with alveolar cells.

We have previously shown that VA10 cells can capture phenotypic architecture of upper and lower airways 
depending cell culture conditions. When cultured in ALI-conditions, VA10 cells can generate pseudostratified epithelium mimicking trachea and larger bronchi and bronchioles. In this model, VA10 generates differentiated ciliated cells as well as goblet cells upon induction. ${ }^{28}$ In addition, we showed when VA10 is embedded into three-dimensional basement membrane matrix in co-culture with endothelial cells, we see increase in branching structures capturing bronchioalveolar phenotype. ${ }^{26}$ Given this plasticity of the basal cell line VA10 and the expression pattern found in IPF lungs, it can be hypothesized that the basal cell could contribute to the fibrotic process in IPF through EMT.

Supplementary Information accompanies the paper on the Laboratory Investigation website (http://www.laboratoryinvestigation.org)

\section{ACKNOWLEDGMENTS}

We thank Professor Deane Mosher for kindly providing us with a fibronectin antibody. Funding was provided by the Icelandic Research Council project (grant number 120423021), the University of Iceland Research- and Doctoral Studies Fund and Landspitali University Hospital Scientific Fund.

\section{DISCLOSURE/CONFLICT OF INTEREST}

The authors declare no conflict of interest.

1. Bradley B, Branley HM, Egan JJ et al. Interstitial lung disease guideline: the British Thoracic Society in collaboration with the Thoracic Society of Australia and New Zealand and the Irish Thoracic Society. Thorax 2008;63:v1-v58.

2. Kim KK, Kugler MC, Wolters PJ et al. Alveolar epithelial cell mesenchymal transition develops in vivo during pulmonary fibrosis and is regulated by the extracellular matrix. Proc Natl Acad Sci USA 2006;103: 13180-13185.

3. Selman $M$, Pardo A. Role of epithelial cells in idiopathic pulmonary fibrosis: from innocent targets to serial killers. Proc Am Thorac Soc 2006:3:364-372.

4. Willis BC, Liebler JM, Luby-Phelps $\mathrm{K}$ et al. Induction of epithelialmesenchymal transition in alveolar epithelial cells by transforming growth factor-beta1: potential role in idiopathic pulmonary fibrosis. Am J Pathol 2005;166:1321-1332.

5. Willis $B C$, duBois RM, Borok Z. Epithelial origin of myofibroblasts during fibrosis in the lung. Proc Am Thorac Soc 2006;3:377-382.

6. Savagner P. The epithelial-mesenchymal transition (EMT) phenomenon. Ann Oncol 2010;21:vii89-vii92.

7. Kerosuo L, Bronner-Fraser M. What is bad in cancer is good in the embryo: importance of EMT in neural crest development. Semin Cell Dev Biol 2012;23:320-332.

8. Nakamura M, Tokura Y. Epithelial-mesenchymal transition in the skin. J Dermatol Sci 2011;61:7-13.

9. Kalluri R, Neilson EG. Epithelial-mesenchymal transition and its implications for fibrosis. J Clin Invest 2003;112:1776-1784.

10. Mongroo PS, Rustgi AK. The role of the miR-200 family in epithelialmesenchymal transition. Cancer Biol Ther 2010;10:219-222.

11. Yang S, Banerjee $S$, de Freitas $A$ et al. Participation of miR-200 in pulmonary fibrosis. Am J Pathol 2012;180:484-493.

12. Eades $G$, Yao $Y$, Yang $M$ et al. miR-200a regulates Sirt1 expression and epithelial to mesenchymal transition (EMT)-like transformation in mammary epithelial cells. J Biol Chem 2011;286:25992-26002.

13. Hilmarsdottir B, Briem E, Bergthorsson JT et al. Functional role of the microRNA-200 family in breast morphogenesis and neoplasia. Genes (Basel) 2014;5:804-820.

14. Korpal M, Lee ES, Hu G et al. The miR-200 family inhibits epithelialmesenchymal transition and cancer cell migration by direct targeting of E-cadherin transcriptional repressors Zeb1 and Zeb2. J Biol Chem 2008;283:14910-14914
15. Rock JR, Barkauskas CE, Cronce MJ et al. Multiple stromal populations contribute to pulmonary fibrosis without evidence for epithelial to mesenchymal transition. Proc Natl Acad Sci USA 2011;108: E1475-E1483.

16. Varma S, Mahavadi P, Sasikumar S et al. Grainyhead-like 2 (Grhl2) distribution reveals novel pathophysiological differences between human idiopathic pulmonary fibrosis and mouse models of pulmonary fibrosis. Am J Physiol Lung Cell Mol Physiol 2014;306:L405-L419.

17. Boers JE, Ambergen AW, Thunnissen FB. Number and proliferation of basal and parabasal cells in normal human airway epithelium. Am J Respir Crit Care Med 1998;157:2000-2006.

18. Rawlins EL, Hogan BL. Epithelial stem cells of the lung: privileged few or opportunities for many? Development 2006;133:2455-2465.

19. Sheikh HA, Fuhrer K, Cieply K et al. P63 expression in assessment of bronchioloalveolar proliferations of the lung. Mod Pathol 2004;17: 1134-1140.

20. Hinata $\mathrm{N}$, Takemura $\mathrm{T}$, Ikushima $\mathrm{S}$ et al. Phenotype of regenerative epithelium in idiopathic interstitial pneumonias. J Med Dent Sci 2003;50:213-224.

21. Das RK, Anura A, Pal M et al. Epithelio-mesenchymal transitional attributes in oral sub-mucous fibrosis. Exp Mol Pathol 2013;95:259-269.

22. Oh JE, Kim $\mathrm{RH}$, Shin $\mathrm{KH}$ et al. Deltanp63alpha protein triggers epithelial-mesenchymal transition and confers stem cell properties in normal human keratinocytes. J Biol Chem 2011;286:38757-38767.

23. Chilosi M, Poletti V, Murer B et al. Abnormal re-epithelialization and lung remodeling in idiopathic pulmonary fibrosis: the role of deltanP63. Lab Invest 2002;82:1335-1345.

24. Seibold MA, Smith RW, Urbanek C et al. The idiopathic pulmonary fibrosis honeycomb cyst contains a mucocilary pseudostratified epithelium. PLoS One 2013;8:e58658.

25. Halldorsson S, Asgrimsson V, Axelsson I et al. Differentiation potential of a basal epithelial cell line established from human bronchial explant. In Vitro Cell Dev Biol Anim 2007;43:283-289.

26. Franzdottir SR, Axelsson IT, Arason AJ et al. Airway branching morphogenesis in three dimensional culture. Respir Res 2010; 11:162.

27. Sigurdsson MI, Isaksson $\mathrm{HJ}$, Gudmundsson $\mathrm{G}$ et al. Diagnostic surgical lung biopsies for suspected interstitial lung diseases: a retrospective study. Ann Thorac Surg 2009;88:227-232.

28. Arason AJ, Jonsdottir HR, Halldorsson $\mathrm{S}$ et al. Deltanp63 has a role in maintaining epithelial integrity in airway epithelium. PLoS One 2014;9: e88683.

29. Saharia A, Guittat L, Crocker S et al. Flap endonuclease 1 contributes to telomere stability. Curr Biol 2008;18:496-500.

30. Godar S, Ince TA, Bell GW et al. Growth-inhibitory and tumorsuppressive functions of p53 depend on its repression of $\mathrm{Cd} 44$ expression. Cell 2008;134:62-73.

31. Carpenter AE, Jones TR, Lamprecht MR et al. Cellprofiler: image analysis software for identifying and quantifying cell phenotypes. Genome Biol 2006:7:R100.

32. Cole BB, Smith RW, Jenkins KM et al. Tracheal basal cells: a facultative progenitor cell pool. Am J Pathol 2010;177:362-376.

33. Kaarteenaho R, Lappi-Blanco E, Lehtonen S. Epithelial N-cadherin and nuclear beta-catenin are up-regulated during early development of human lung. BMC Dev Biol 2010;10:113.

34. Yamaya M, Finkbeiner WE, Chun SY et al. Differentiated structure and function of cultures from human tracheal epithelium. Am J Physiol 1992;262:L713-L724.

35. Sachs LA, Finkbeiner WE, Widdicombe JH. Effects of media on differentiation of cultured human tracheal Epithelium. In Vitro Cell Dev Biol Anim 2003;39:56-62.

36. Boyer B, Tucker GC, Valles AM et al. Reversible transition towards a fibroblastic phenotype in a rat carcinoma cell line. Int J Cancer Suppl 1989;4:69-75.

37. Hunter I, Lindh $\mathrm{M}$, Obrink B. Differential regulation of C-cam isoforms in epithelial cells. J Cell Sci 1994;107(Pt 5): 1205-1216.

38. Turner R, Lozoya $\mathrm{O}$, Wang $\mathrm{Y}$ et al. Human hepatic stem cell and maturational liver lineage biology. Hepatology 2011;53:1035-1045.

39. Munz M, Baeuerle PA, Gires $O$. The emerging role of EpCAM in cancer and stem cell signaling. Cancer Res 2009;69:5627-5629.

40. Santisteban M, Reiman JM, Asiedu MK et al. Immune-induced epithelial to mesenchymal transition in vivo generates breast cancer stem cells. Cancer Res 2009;69:2887-2895. 
41. Frederick BA, Helfrich BA, Coldren CD et al. Epithelial to mesenchyma transition predicts gefitinib resistance in cell lines of head and neck squamous cell carcinoma and non-small cell lung carcinoma. Mol Cancer Ther 2007;6:1683-1691.

42. Crespin S, Bacchetta M, Bou Saab J et al. Cx26 regulates proliferation of repairing basal airway epithelial cells. Int J Biochem Cell Biol 2014;52: 152-160.

43. Kropski JA, Lawson WE, Young LR et al. Genetic studies provide clues on the pathogenesis of idiopathic pulmonary fibrosis. Dis Models Mech 2013;6:9-17.

44. Nogee LM, Dunbar 3rd AE, Wert SE et al. A mutation in the surfactant protein $\mathrm{C}$ gene associated with familial interstitial lung disease. $\mathrm{N}$ Engl J Med 2001;344:573-579.

45. Wang Y, Kuan PJ, Xing $C$ et al. Genetic defects in surfactant protein A2 are associated with pulmonary fibrosis and lung cancer. Am J Hum Genet 2009;84:52-59.

46. Armanios MY, Chen JJ, Cogan JD et al. Telomerase mutations in families with idiopathic pulmonary fibrosis. N Engl J Med 2007:356:1317-1326.

47. Tsakiri KD, Cronkhite JT, Kuan PJ et al. Adult-onset pulmonary fibrosis caused by mutations in telomerase. Proc Natl Acad Sci USA 2007;104:7552-7557.

48. Seibold MA, Wise AL, Speer $M C$ et al. A common Muc5b promoter polymorphism and pulmonary fibrosis. N Engl J Med 2011;364:1503-1512.

49. Fingerlin TE, Murphy E, Zhang W et al. Genome-wide association study identifies multiple susceptibility loci for pulmonary fibrosis. Nat Genet 2013;45:613-620.
50. Hunninghake GM, Hatabu $\mathrm{H}$, Okajima $\mathrm{Y}$ et al. Muc5b promoter polymorphism and interstitial lung abnormalities. $\mathrm{N}$ Engl J Med 2013:45:613-620.

51. Korfei M, Ruppert C, Mahavadi P et al. Epithelial endoplasmic reticulum stress and apoptosis in sporadic idiopathic pulmonary fibrosis. Am J Respir Crit Care Med 2008;178:838-846.

52. Armanios M, Blackburn EH. The telomere syndromes. Nat Rev Genet 2012;13:693-704

53. Lawson WE, Cheng DS, Degryse AL et al. Endoplasmic reticulum stress enhances fibrotic remodeling in the lungs. Proc Natl Acad Sci USA 2011;108:10562-10567.

54. Tanjore $\mathrm{H}$, Blackwell TS, Lawson WE. Emerging evidence for endoplasmic reticulum stress in the pathogenesis of idiopathic pulmonary fibrosis. Am J Physiol Lung Cell Mol Physiol 2012;302: L721-L729.

55. Zhong Q, Zhou B, Ann DK et al. Role of endoplasmic reticulum stress in epithelial-mesenchymal transition of alveolar epithelial cells: effects of misfolded surfactant protein. Am J Respir Cell Mol Biol 2011;45: 498-509.

56. Thai $\mathrm{P}$, Loukoianov A, Wachi S et al. Regulation of airway mucin gene expression. Annu Rev Physiol 2008;70:405-429.

57. Buckley ST, Medina C, Ehrhardt C. Differential susceptibility to epithelial-mesenchymal transition (EMT) of alveolar, bronchial and intestinal epithelial cells in vitro and the effect of angiotensin II receptor inhibition. Cell Tissue Res 2010;342:39-51. 